\title{
Continuous-variable quantum cryptography is secure against non-gaussian attacks
}

\author{
Frédéric Grosshans and Nicolas J. Cerf \\ Quantum Information and Communication, École Polytechnique, \\ CP 165, Université Libre de Bruxelles, B-1050 Brussels, Belgium
}

(Dated: August 20, 2018)

\begin{abstract}
A general study of arbitrary finite-size coherent attacks against continuous-variable quantum cryptographic schemes is presented. It is shown that, if the size of the blocks that can be coherently attacked by an eavesdropper is fixed and much smaller than the key size, then the optimal attack for a given signal-to-noise ratio in the transmission line is an individual gaussian attack. Consequently, non-gaussian coherent attacks do not need to be considered in the security analysis of such quantum cryptosystems.

PACS numbers: 03.67.Dd, 42.50.-p, 89.70.+c

Keywords: Quantum cryptography, Continuous variables, Security, Finite-size non-Gaussian coherent attacks
\end{abstract}

Continuous-variable quantum information theory has attracted a rapidly increasing interest over the past few years (see, e.g., [1]). In this context, several quantum key distribution (QKD) schemes based on the exchange of continuous key carriers have been proposed (see, e.g., 2]). In particular, several schemes based on the continuous modulation of coherent or squeezed states of light supplemented with homodyne detection have been shown to be particularly efficient for distributing secret keys at high repetition rates [3, 4]. An experimental demonstration of key distribution based on a gaussian modulation of coherent states was recently provided in [5].

In this Letter, we prove that given the estimated covariance matrix of Alice's and Bob's data, the optimal finite-size coherent attack reduces to an individual gaussian attack characterized by this covariance matrix. This result fundamentally originates from the property that the distribution maximizing its Shannon entropy for a given variance is a gaussian distribution. This, combined with an entropic uncertainty relation, implies that is is sufficient to check the security of such cryptosystems against the restricted class of gaussian attacks. In other words, the best strategy for Eve is to apply sequentially, on each key element, a gaussian cloning machine $[\underline{6}$ or an entangling gaussian cloning machine [7] depending on the exact protocol used. Another consequence is that, in order to maximize the resulting secret key rate via the gaussian channel induced by Eve's attack, Alice should modulate her data with a gaussian distribution.

The security proof presented here is valid for all continuous-variable QKD schemes where Alice and Bob monitor the transmission line via the second-order moments of their data, which includes all the protocols considered in our previous papers $[3,4,5]$. Note, however, that this excludes the alternative protocol based on postselection as presented in [8]. Our proof covers all possible (including coherent) attacks that an eavesdropper may apply on finite-size blocks of key elements. The block size may be arbitrary, but it must be much smaller than the key size, so that the key is made out of a large number of independent blocks and statistical arguments therefore warrant the use of information theory in the proof. The unconditional security of squeezed-state QKD against coherent attacks is currently proven if the squeezing exceeds some threshold 11], while such a proof for coherent-state QKD is the topic of a separate study [12].

Squeezed state protocols. Let us first investigate the security of gaussian-modulated squeezed-state protocols [3]. Alice chooses a quadrature $(q$ or $p)$ at random and sends Bob a displaced squeezed state, where the squeezing and displacement are applied on the chosen quadrature while the value of the displacement is gaussian distributed. After transmission via the quantum channel, which may be controlled by Eve, Bob then measures $q$ or $p$ at random. After disclosing the quadrature they used, Alice and Bob discard their data when the quadratures differ, while the rest is used to make a secret key 9, 10]. We will in fact consider equivalent entanglementbased protocols [4], where Alice prepares a two-mode vacuum squeezed state, measures a quadrature of one of the beams and sends the other beam to Bob. Alice and Bob iterate these actions $n$ times, while we assume that Eve is able to apply some arbitrary joint operation on this block of $n$ pulses. In order to acquire accurate statistics, Alice and Bob repeat this protocol $L$ times (with $L \gg 1$ ), that is, they exchange $L$ blocks of $n$ pulses in total. In our security analysis below, we will apply information theory at the level of blocks, which is justified since $L \gg 1$.

We model Eve's attack by considering that Alice, Bob, and Eve share a pure tripartite entangled state (see Fig. 11. Alice's (resp. Bob's) part of the state is a set of $n$ modes, denoted by A (resp. B). The unknown physical system kept by Eve is denoted by $E$. The joint state is pure since we must assume that Eve is able to control the environment, thereby to purify the state. We also suppose that Bob always measures the same quadrature $Q$ as Alice $(Q=q$ or $p)$. This requires the availability of a quantum memory (Bob delays his measurement until 


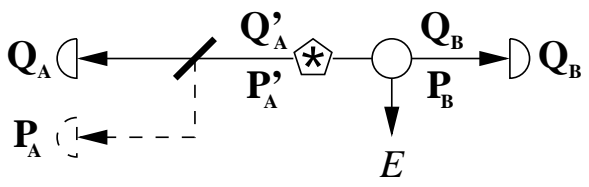

FIG. 1: Equivalent entanglement-based QKD protocol. The twin beams of an EPR-source $\left(^{*}\right)$ are sent to homodyne detectors at Alice's (left) and Bob's (right) side. In the analogue of the squeezed-state protocol, the beam-splitter and the dashed lines are omitted, so Alice only measures one quadrature $\left(\mathbf{Q}_{\mathbf{A}^{\prime}}=\mathbf{Q}_{\mathbf{A}}\right)$. In the analogue of the coherentstate protocol, the beam-splitter is used by Alice to measure $\mathbf{Q}_{\mathbf{A}^{\prime}}$ and $\mathbf{P}_{\mathbf{A}^{\prime}}$ simultaneously [13].

Alice discloses the quadrature she used). In a more realistic scheme where Alice and Bob independently choose their quadrature $q$ or $p$ at random, they agree only half of the time, which simply leads to a factor $1 / 2$ in the information rates computed below.

Information Rates. The mutual information between Alice's and Bob's data is

$$
I(\mathbf{B} ; \mathbf{A})=I\left(\mathbf{Q}_{\mathbf{B}} ; \mathbf{Q}_{\mathbf{A}}\right)=H\left(\mathbf{Q}_{\mathbf{B}}\right)-H\left(\mathbf{Q}_{\mathbf{B}} \mid \mathbf{Q}_{\mathbf{A}}\right),
$$

where $\mathbf{Q}_{\mathbf{A}}$ (resp. $\mathbf{Q}_{\mathbf{B}}$ ) is the random vector of Alice's (resp. Bob's) measured quadratures on a block of $n$ pulses, while $H(\cdot)$ [resp. $H(\cdot \mid \cdot)$ ] denotes the Shannon entropy (resp. conditional entropy) for continuous random variables. We focus our attention on reverse reconciliation protocols [5, 7], in which Bob's data are used to make the key instead of Alice's data (direct reconciliation). Then, Eve tries to get the maximum information on Bob's measurement outcomes $\mathbf{Q}_{\mathbf{B}}$ through a measurement of her ancilla $E$ (we denote Eve's ancilla and her measurement outcomes by the same symbol $E$ ). Eve's information is

$$
I(\mathbf{B} ; E)=I\left(\mathbf{Q}_{\mathbf{B}} ; E\right)=H\left(\mathbf{Q}_{\mathbf{B}}\right)-H\left(\mathbf{Q}_{\mathbf{B}} \mid E\right) .
$$

The secret key rate Alice and Bob are guaranteed to be able to distill by reverse reconciliation is $[14,15]$

$$
\begin{aligned}
\Delta I & =I(\mathbf{B} ; \mathbf{A})-I(\mathbf{B} ; E) \\
& =H\left(\mathbf{Q}_{\mathbf{B}} \mid E\right)-H\left(\mathbf{Q}_{\mathbf{B}} \mid \mathbf{Q}_{\mathbf{A}}\right) .
\end{aligned}
$$

Alice and Bob can, in principle, estimate $H\left(\mathbf{Q}_{\mathbf{B}} \mid \mathbf{Q}_{\mathbf{A}}\right)$ with arbitrary precision since they have access to $L$ joint realizations of the random vectors $\mathbf{Q}_{\mathbf{A}}$ and $\mathbf{Q}_{\mathbf{B}}$. To lower bound Eve's uncertainty on the key $H\left(\mathbf{Q}_{\mathbf{B}} \mid E\right)$, they can use the entropic uncertainty relation that applies to the two sets of conjugate quadratures $\mathbf{Q}_{\mathbf{B}}$ and $\mathbf{P}_{\mathbf{B}}[16$, 17]. Indeed, we know that by measuring their systems, Alice and Eve project Eve's system onto a pure state since the three of them share a joint pure state. Thus, conditionally on Alice's and Eve's measurements $\mathbf{P}_{\mathbf{A}}$ and $E$, the pure state held by Bob must satisfy the entropic inequality

$$
H\left(\mathbf{Q}_{\mathbf{B}} \mid E\right)+H\left(\mathbf{P}_{\mathbf{B}} \mid \mathbf{P}_{\mathbf{A}}\right) \geq 2 n H_{0},
$$

where $H_{0}$ is the entropy of a quadrature of the vacuum state for an harmonic oscillator. This inequality then allows us to lower bound the accessible secret key rate regardless the action of Eve, namely

$$
\Delta I \geq \Delta I_{\min }=2 n H_{0}-H\left(\mathbf{Q}_{\mathbf{B}} \mid \mathbf{Q}_{\mathbf{A}}\right)-H\left(\mathbf{P}_{\mathbf{B}} \mid \mathbf{P}_{\mathbf{A}}\right) .
$$

It is worth stressing that the random vectors $\mathbf{P}_{\mathbf{A}}$ and $\mathbf{P}_{\mathbf{B}}$ denote the quadratures that could have been measured (the measured quadratures are $\mathbf{Q}_{\mathbf{A}}$ and $\mathbf{Q}_{\mathbf{B}}$ ). These quadratures are, of course, not directly accessible, but we only need their statistical distribution here in order to upper bound Eve's information. This distribution can be estimated from the other pulses for which the measured quadrature is the same as $\mathbf{P}_{\mathbf{A}}$ and $\mathbf{P}_{\mathbf{B}}$. For simplicity, we assume that the two physical quadratures $q$ and $p$ are both chosen with probability $1 / 2$. This implies that $\mathbf{Q}_{\mathbf{A}}$ and $\mathbf{P}_{\mathbf{A}}$ play fully identical roles so they can be treated completely symmetrically (the same is true for $\mathbf{Q}_{\mathbf{B}}$ and $\left.\mathbf{P}_{\mathbf{B}}\right)$. We insist on that this symmetry is not a limitation on Eve's possible actions. Even if Eve has a quantum memory and acts differently on the physical quadratures $q$ and $p$ (after the selected quadrature is disclosed), each of them has an equal probability to be a measured $\left(\mathbf{Q}_{\mathbf{A}}\right.$ and $\left.\mathbf{Q}_{\mathbf{B}}\right)$ or an unmeasured $\left(\mathbf{P}_{\mathbf{A}}\right.$ and $\mathbf{P}_{\mathbf{B}}$ ) quadrature. Since Eve has no way of guessing which physical quadrature is used, this symmetry imposes $H\left(\mathbf{Q}_{\mathbf{B}} \mid \mathbf{Q}_{\mathbf{A}}\right)=H\left(\mathbf{P}_{\mathbf{B}} \mid \mathbf{P}_{\mathbf{A}}\right)=H(\mathbf{B} \mid \mathbf{A})$, where we now use $\mathbf{A}$ and $\mathbf{B}$ as a shorthand notation for $\mathbf{Q}_{\mathbf{A}}$ and $\mathbf{Q}_{\mathbf{B}}\left(\right.$ or $\mathbf{P}_{\mathbf{A}}$ and $\left.\mathbf{P}_{\mathbf{B}}\right)$. Therefore

$$
\Delta I_{\min }=2\left(n H_{0}-H(\mathbf{B} \mid \mathbf{A})\right) .
$$

Since Alice and Bob can evaluate $H(\mathbf{B} \mid \mathbf{A})$ by statistical sampling, they get an estimate of $\Delta I_{\min }$ and can use relevant algorithms to extract a secret key with at least this rate $[9,10]$.

Individual attacks are optimal. We first prove that Alice and Bob can lower bound $\Delta I_{\min }$ simply by assuming that Eve performs an individual attack. Let $A_{i}$ (resp. $B_{i}$ ) be the $i$ th component of the random vector $\mathbf{A}$ (resp. B). The subadditivity of Shannon entropy implies that

$$
H(\mathbf{B} \mid \mathbf{A}) \leq \sum_{i} H\left(B_{i} \mid \mathbf{A}\right)
$$

while each term of the summation can be bounded by use of the strong subadditivity of the entropy, namely

$$
H\left(B_{i} \mid \mathbf{A}\right)=H\left(B_{i} \mid A_{1}, \ldots, A_{n}\right) \leq H\left(B_{i} \mid A_{i}\right),
$$

so that

$$
H(\mathbf{B} \mid \mathbf{A}) \leq \sum_{i} H\left(B_{i} \mid A_{i}\right)
$$

We now consider the average joint distribution of $\mathrm{Al}-$ ice's and Bob's measurement outcomes (averaged over 
the block of size $n$ ). Suppose that $A$ and $B$ are distributed according to a mixture of the $A_{i}$ 's and $B_{i}$ 's, with the index $i$ being randomly drawn from a uniform distribution, that is

$$
\mathcal{P}(A=a, B=b)=\frac{1}{n} \sum_{i} \mathcal{P}\left(A_{i}=a, B_{i}=b\right), \quad \forall a, b .
$$

Then, the strong subadditivity of entropies implies that

$$
H\left(B_{i} \mid A_{i}\right)=H(B \mid A, i) \leq H(B \mid A),
$$

so that Eq. (9) transforms into

$$
H(\mathbf{B} \mid \mathbf{A}) \leq n H(B \mid A) .
$$

Finally, using Eq. (6), one gets

$$
\Delta I_{\min } \geq 2 n\left(H_{0}-H(B \mid A)\right) .
$$

This means that, to be safe against finite-size coherent attacks, Alice and Bob only need to evaluate $H(B \mid A)$, a conditional entropy for a distribution in $\mathbb{R}^{2}$, instead of $H(\mathbf{B} \mid \mathbf{A})$, a conditional entropy for a distribution in $\mathbb{R}^{2 n}$.

To better understand this conclusion, assume that Eve applies a coherent attack which induces correlations between the various components of $\mathbf{A}$ and $\mathbf{B}$ inside each block. These correlations force Eve to induce a kind of structure in Alice's and Bob's data, which would not be present for individual attacks, so Eve is actually limiting herself. Overlooking these correlations and considering individual attacks only may be suboptimal for Alice and Bob when estimating $\Delta I_{\text {min }}$, but it guarantees they are on the safe side.

Gaussian attacks are optimal. Now, we prove that $H(B \mid A)$ can be upper bounded simply by measuring the covariance matrix $\mathbf{K}$ of variables $A$ and $B$,

$$
\mathbf{K}=\left[\begin{array}{cc}
\left\langle A^{2}\right\rangle & \langle A B\rangle \\
\langle A B\rangle & \left\langle B^{2}\right\rangle
\end{array}\right]
$$

which is much easier than estimating $H(B \mid A)$. To simplify the notations, we will assume that $\langle A\rangle=\langle B\rangle=0$ (in practice, this should be checked and possibly corrected by applying the adequate shift). For a given $\mathbf{K}$, if Alice knows $A$, her linear estimate of $B$ that minimizes the error variance is given by $\frac{\langle A B\rangle}{\left\langle A^{2}\right\rangle} A$. Denoting by $\delta B$ the error of this best linear estimate,

$$
\delta B=B-\frac{\langle A B\rangle}{\left\langle A^{2}\right\rangle} A
$$

we have

$$
H(B \mid A)=H(\delta B \mid A) \leq H(\delta B) .
$$

where we have used the translation invariance and the subadditivity of Shannon entropy. Since the gaussian distribution has the maximum entropy for a given variance, one has

$$
H(\delta B) \leq H_{\mathcal{G}}(\delta B)
$$

where $H_{\mathcal{G}}(\delta B)$ is the entropy of a gaussian distribution having the variance $\left\langle\delta B^{2}\right\rangle=\left\langle B^{2}\right\rangle-\langle A B\rangle^{2} /\left\langle A^{2}\right\rangle$. In the case where $A$ and $B$ are drawn from an equivalent bivariate Gaussian distribution with the same covariance matrix $\mathbf{K}$ as the observed distribution, we note that $\delta B$ and $A$ become uncorrelated, so that

$$
H_{\mathcal{G}}(\delta B)=H_{\mathcal{G}}(\delta B \mid A)
$$

Chaining Eqs. (16) to (18) and using the translation invariance of entropy, one obtains

$$
H(B \mid A) \leq H_{\mathcal{G}}(B \mid A),
$$

which, combined with Eq. (13), yields

$$
\Delta I_{\min } \geq 2 n\left(H_{0}-H_{\mathcal{G}}(B \mid A)\right) .
$$

Finally, the conditional entropy $H_{\mathcal{G}}(B \mid A)$ of a bivariate gaussian distribution being a simple function of $\mathbf{K}$, one obtain the central result of this paper,

$$
\Delta I_{\min } \geq n \log \frac{N_{0}}{\left\langle\delta B^{2}\right\rangle},
$$

where $N_{0}$ represents the vacuum variance. This expression coincides with the one found when limiting Eve to gaussian individual attacks [4, 5]. Therefore, the optimal attack given the observed covariance matrix $\mathbf{K}$ is a gaussian individual attack as described in [5, 7, 13].

The optimality of gaussian attacks can be interpreted almost alike the optimality of individual attacks : since the gaussian distribution has the maximal entropy, nongaussian attacks are more structured than gaussian ones for a same added noise variance, so Eve is more restricted. Therefore, if Alice and Bob only monitor the covariance matrix $\mathbf{K}$, they can safely assume that Eve uses gaussian attacks. If Eve indeed applies a gaussian attack, the best Alice and Bob can do is to use independent and gaussiandistributed key elements, which saturates all the involved inequalities, so that $\Delta I_{\min }$ is the highest. This justifies a posteriori the choice of gaussian-modulated QKD protocols in [3, 4, 5].

Coherent state protocols. We now extend the proof to QKD protocols based on gaussian-modulated coherent states [4, 5, 7]. We again exploit the property that these protocols are equivalent to some entanglement-based protocols where Alice jointly measures $q$ and $p$ on her entangled beam while sending the other one to Bob [13]. The central point is that this "virtual entanglement," which may have existed between Alice and Bob, must be taken into account when bounding Eve's information even if the actual protocol makes no use of entanglement. We will denote by $\mathbf{Q}_{\mathbf{A}^{\prime}}$ and $\mathbf{P}_{\mathbf{A}^{\prime}}$ the vectors of the two quadratures of the $n$ beams kept by Alice and $\mathbf{Q}_{\mathbf{B}}$ the vector of the $n$ quadratures measured by Bob (see Fig. (1). The difference with the previous scheme is that Alice attempts to measure simultaneously $\mathbf{Q}_{\mathbf{A}^{\prime}}$ and $\mathbf{P}_{\mathbf{A}^{\prime}}$ through a 50:50 
beam-splitter followed by two homodyne detectors. The measurement outcomes $\mathbf{Q}_{\mathbf{A}}$ and $\mathbf{P}_{\mathbf{A}}$ suffer from added noise, while Alice never has access to the actual values $\mathbf{Q}_{\mathbf{A}^{\prime}}$ and $\mathbf{P}_{\mathbf{A}^{\prime}}$. The expression of $H\left(\mathbf{Q}_{\mathbf{B}} \mid \mathbf{Q}_{\mathbf{A}}\right)$ only depends on measured quantities so it can be statistically estimated as before, but the entropic uncertainty relation used to bound $H\left(\mathbf{Q}_{\mathbf{B}} \mid E\right)$ now involves the physical beam on Alice's side, so one has

$$
H\left(\mathbf{Q}_{\mathbf{B}} \mid E\right)+H\left(\mathbf{P}_{\mathbf{B}} \mid \mathbf{P}_{\mathbf{A}^{\prime}}\right) \geq 2 n H_{0}
$$

Thus, the same reasoning as before now leads to

$$
\Delta I_{\min } \geq n\left(2 H_{0}-H_{\mathcal{G}}(B \mid A)-H_{\mathcal{G}}\left(B \mid A^{\prime}\right)\right),
$$

where $H_{\mathcal{G}}\left(B \mid A^{\prime}\right)$ is the conditional entropy of a gaussian distribution having the same covariance matrix $\mathbf{K}^{\prime}$ than $A^{\prime}$ and $B$, which is

$$
\mathbf{K}^{\prime}=\left[\begin{array}{cc}
\left\langle A^{\prime 2}\right\rangle & \left\langle A^{\prime} B\right\rangle \\
\left\langle A^{\prime} B\right\rangle & \left\langle B^{2}\right\rangle
\end{array}\right]=\left[\begin{array}{cc}
2\left(\left\langle A^{2}\right\rangle-N_{0}\right) & \sqrt{2}\langle A B\rangle \\
\sqrt{2}\langle A B\rangle & \left\langle B^{2}\right\rangle
\end{array}\right]
$$

One has therefore

$$
\Delta I_{\min } \geq n \log \frac{N_{0}}{\sqrt{\left(\left\langle B^{2}\right\rangle-\frac{\langle A B\rangle^{2}}{\left\langle A^{2}\right\rangle}\right)\left(\left\langle B^{2}\right\rangle-\frac{\langle A B\rangle^{2}}{\left\langle A^{\prime 2}\right\rangle-N_{0}}\right)}},
$$

which is exactly the same expression as in our previous papers [5, 7, 13], where the only considered attacks are gaussian individual attacks.

Discussion. We extended to finite-size non-gaussian attacks the validity of the previous security proofs for continuous-variable QKD schemes when Eve's intervention is bounded via the measured added noise variance in the channel. Our proof focuses on the schemes based on reverse reconciliation since these are known to tolerate larger losses than the direct reconciliation-based protocols in the case of gaussian individual attacks. Adapting the proof to direct-reconciliation [3, 4] or even other [8] protocols will treated elsewhere. In the proof, we assume the protocol is ideal, that is, a perfect one-way reconciliation algorithm is available. However, realistic reconciliation protocols are imperfect 10]: the number of correlated bits that can be extracted from Alice's and Bob's data never attains Shannon's limit $I(B ; A)$ and may become low if Eve's attack has an unexpected shape, the reconciliation protocol being adapted to a specific noise structure. Nevertheless, the security proof can be easily extended to this situation since Alice and Bob can always compute the effective value of their shared information $I_{\text {eff }}$ by comparing subsets of their data. Then, using $I(B ; E) \leq n\left(H_{0}-H_{G}\left(B \mid A^{\prime}\right)\right)$ as before, one obtains $\Delta I_{\mathrm{min}} \geq I_{\mathrm{eff}}-I(B ; E)$.

Finally, we have shown that there is a fundamental link between security and "entropic squeezing": the security is guaranteed $\left(\Delta I_{\min }>0\right)$ if the conditional entropy $H(B \mid A)$ is below the quantum limit $H_{0}$ [Eq. (13)]. In the gaussian case, this simplifies to condition $\sigma^{2}(B \mid A)<N_{0}$ [Eq. (21)], where $\sigma^{2}(B \mid A)=\left\langle\delta B^{2}\right\rangle$ denotes the conditional variance of $B$ knowing $A$, as suggested in [18]. The latter condition is, however, over-pessimistic if Eve uses a non-gaussian attack, since $\sigma^{2}(B \mid A)$ might exceed $N_{0}$, destroying the conditional squeezing, while keeping $H(B \mid A)$ low enough to ensure security. If Alice and Bob only monitor the covariance matrix $\mathbf{K}$, this attack is non-optimal since the worst-case gaussian attack would maximize $H(B \mid A)$ and thereby minimize $\Delta I$ for a given $\sigma^{2}(B \mid A)$. In conclusion, the security can be warranted by requiring conditional squeezing, which is more stringent than entropic squeezing but much easier to assess.

We are grateful to Philippe Grangier for stimulating this work, and to Patrick Navez and Gilles Van Assche for useful discussions. FG acknowledges support from the Belgian National Fund for Scientific Research. NJC acknowledges financial support from the Communauté Française de Belgique under grant ARC 00/05-251, from the IUAP programme of the Belgian governement under grant V-18, and from the EU under project RESQ (IST2001-35759).

[1] S.L. Braunstein and A.K. Pati, Quantum information theory with continuous variables, (Kluwer Academic, Dordrecht, 2003).

[2] M. Hillery, Phys. Rev. A 61, 022309 (2000).

[3] N.J. Cerf, M. Lévy, and G. Van Assche, Phys. Rev. A 63, 052311 (2001).

[4] F. Grosshans and Ph. Grangier, Phys. Rev. Lett. 88, 057902 (2002).

[5] F. Grosshans, G. Van Assche, J. Wenger, R. Brouri, N.J. Cerf, and Ph. Grangier, Nature (London) 421, 238 (2003).

[6] N.J. Cerf, A. Ipe, and X. Rottenberg, Phys. Rev. Lett. 85, 1754 (2000).

[7] F. Grosshans and Ph. Grangier, Proc. 6th Int. Conf. on Quantum Communications, Measurement, and Computing, (Rinton Press, Princeton, 2003); quant-ph/0204127

[8] Ch. Silberhorn, T. C. Ralph, N. Lütkenhaus, and G. Leuchs, Phys. Rev. Lett. 89, 167901 (2002).

[9] G. Van Assche, J. Cardinal, and N.J. Cerf, arXiv cs.CR/0107030 IEEE Trans. Inform. Theory, in press.

[10] On-line supplementary information of Ref. [5].

[11] D. Gottesman and J. Preskill, Phys. Rev. A 63, 022309 (2001).

[12] S. Iblisdir, G. Van Assche, and N. J. Cerf, unpublished.

[13] F. Grosshans, N. J. Cerf, J. Wenger, R. Brouri, and Ph. Grangier, arXiv quant-ph/0306141 Q.I.C., in press.

[14] I. Csiszar and J. Korner, IEEE Trans. Inform. Theory 24, 339 (1978).

[15] U. Maurer, IEEE Trans. Inform. Theory 39, 733 (1993).

[16] I. Bialynicki-Birula and J. Mycielski, Commun. Math. Phys. 44, 129 (1975).

[17] W. Beckner, Ann. Math. 102, 159 (1975).

[18] F. Grosshans and Ph. Grangier, Phys. Rev. A 64, 010301(R). 